\title{
Estilos de vida y síndrome metabólico en adultos y adultos mayores de Trujillo, Perú, 2019
}

\author{
Lifestyles and metabolic syndrome in the adult and elderly population from \\ Trujillo, Peru, 2019 \\ Jordano Jackestuard Ismael Carranza Paredes ${ }^{1, a}$, Jorge Luis Díaz-Ortega « 1,b, Rosa Patricia Gálvez Carrillo 1,c
}

Filiación y grado académico

Universidad César Vallejo, Trujillo, Perú

a Bachiller del Programa Académico de Nutrición.

Doctor en Ciencias Biomédicas, docente del Programa Académico de Nutrición.

Doctor en Ciencias Ambientales, docente del Programa Académico de Nutrición.

(iD) ORCID iD de Jordano Carranza Paredes https://orcid.org/0000-0003-4367-3136

(iD) ORCID iD de Jorge Luis Díaz-Ortega https://orcid.org/0000-0002-6154-8913

(iD) ORCID iD de Rosa Gálvez Carrillo https://orcid.org/0000-0002-4612-109X

Contribución de autoría

Todos los autores contribuyeron en el diseño, ejecución, redacción y revisión del artículo.

Fuentes de financiamiento

Autofinanciado.

Conflictos de interés

Los autores declaramos no presentar ningún conflicto de interés.

Recibido: 05-04-2021

Arbitrado por pares

Aceptado: 12-07-2021

Citar como

Carranza Paredes JI, Díaz-Ortega JL, Gálvez Carrillo RP. Estilos de vida y síndrome metabólico en adultos y adultos mayores de Trujillo, Perú, 2019. Rev Peru Cienc Salud. 2021; 3(3): 164-72. doi: https://doi.org/10.37711/ rpcs.2021.3.3.336

Correspondencia Jorge Díaz-Ortega

Dirección: Mz. I'Lote 1 Urb. Vista Hermosa Distrito de Trujillo, Departamento de la Libertad, Perú.

Email: jdiazo@ucv.edu.pe

\section{RESUMEN}

Objetivo. Determinar la relación de los estilos de vida y el síndrome metabólico en los pobladores adultos y adultos mayores de la ciudad de Trujillo (Perú), entre julio y agosto de 2019. Métodos. El estudio fue de diseño no experimental, descriptivo, correlacional y de corte transversal. La muestra estuvo conformada por 227 adultos y 44 adultos mayores. El síndrome metabólico se determinó mediante criterios del ATP-III armonizado. Para evaluar los estilos de vida se utilizó un cuestionario con preguntas sobre snacks, frutas, verduras, consumo de tabaco, alcohol y actividad física. El análisis estadístico empleado para relacionar ambas variables fue la prueba de chi cuadrado. Resultados. Se determinó que el $55 \%$ de los adultos y el $73 \%$ de los adultos mayores presentaban síndrome metabólico. El 18,9\% de los adultos consumían snacks y presentaban síndrome metabólico; en tanto que aquellos que no consumían snacks y no presentaban síndrome metabólico representaban el $23,3 \%$ ( $p=0,037)$. El $27,3 \%$ no consumían verduras, presentaban síndrome metabólico; en tanto que el $28,6 \%$ consumían verduras, no presentaban síndrome metabólico $(0,044)$. Conclusión. Solo se encontró relación entre el consumo de snacks y el consumo de verduras con el síndrome metabólico en los adultos.

Palabras clave: estilo de vida; síndrome metabólico; adulto; snacks (fuente: DeCS-BIREME).

\section{ABSTRACT}

Objective. To determine the relationship of lifestyles and metabolic syndrome in the adult and elderly population in the city of Trujillo (Peru), between July and August 2019. Methods. The study was nonexperimental, descriptive, correlational and cross-sectional. The sample consisted of 227 adults and 44 elderlies. Metabolic syndrome was determined by criteria of harmonised ATP-III. To evaluate lifestyles, a questionnaire about snacks, fruits, vegetables, tobacco consumption, alcohol and physical activity was used. The statistical analysis used to relate both variables was the chi-square test. Results. It was found that $55 \%$ of adults and $73 \%$ of elderlies had metabolic syndrome. $18.9 \%$ of adults consumed snacks and had metabolic syndrome; while those who did not consume snacks and did not have metabolic syndrome represented $23.3 \%$ ( $p=0.037$ ). $27.3 \%$ did not consume vegetables, they had metabolic syndrome; while $28.6 \%$ consumed vegetables, they did not have metabolic syndrome (0.044). Conclusion. Only the consumption of snacks and the consumption of vegetables were related to metabolic syndrome in adults.

Keywords: lifestyle; metabolic syndrom; adult; snacks (source: MeSH NLM). 


\section{INTRODUCCIÓN}

Existe un aumento global en la prevalencia de las enfermedades cardiovasculares y el síndrome metabólico, el cual ha contribuido en el incremento significativo de la morbilidad. La prevalencia global de las enfermedades cardiovasculares se calcula que afecta a 422,7 millones de la población mundial( ${ }^{(1)}$.

El concepto de síndrome metabólico fue introducido para facilitar la identificación de la población con alto riesgo de desarrollar enfermedades de riesgo cardiovascular. El síndrome metabólico involucra aspectos de riesgo como el incremento de la circunferencia abdominal, glicemia elevada, hipertensión, niveles bajos de lipoproteína de alta densidad (HDL) e hipertrigliceridemia ${ }^{(2,4)}$. Según las estimaciones de la Federación Internacional de Diabetes (FID), alrededor de uno de cada cuatro individuos en todo el mundo se ven afectados por el síndrome metabólico, con posibles diferencias según la edad, el sexo y el origen étnico de la población en estudio ${ }^{(5,6)}$.

En un estudio desarrollado en cinco estratos de la población peruana se ha determinado una prevalencia de síndrome metabólico en $25,1 \%$ de la población ${ }^{(7)}$; así mismo Linares et al., en el año 2014 reportaron que en la ciudad de Trujillo la prevalencia era $22 \%{ }^{(8)}$.

Se indica una relación estadística entre el consumo de alcohol y el síndrome metabólico, independientemente del tipo de alcohol consumido y en todos los niveles de consumo, con reducción del HDL, incremento en las cifras de glucemia, de triglicéridos y de tensión arterial, siendo estos efectos más marcados si se añade presencia previa de sobrepeso u obesidad ${ }^{(9)}$.

En relación al tabaquismo, fumar un cigarrillo significa exponerse a la acción de los principios activos como nicotina, alquitrán y monóxido de carbono (CO), entre otros, en cantidades significativas, lo que favorece el avance rápido de la aterosclerosis y da lugar al envejecimiento prematuro, anticipa la senilidad y acorta la esperanza de vida ${ }^{(10)}$.

Actualmente, se está produciendo una transición epidemiológica de enfermedades predominantemente transmisibles y deficiencias nutricionales a enfermedades principalmente no transmisibles que se puede atribuir al aumento de la urbanización y los estilos de vida cambiantes, con el avance de las economías que conduce a dietas aterogénicas en la población, reducción de la actividad física, aumento del tabaquismo, aumento del consumo de alcohol y, por tanto, en altos niveles de obesidad ${ }^{(11)}$.
Por esta razón se consideró como objetivos identificar los estilos de vida y la presencia del síndrome metabólico en adultos y adultos mayores de la ciudad de Trujillo para, seguidamente, establecer la relación entre dichas variables. Este estudio permitirá establecer estrategias de orientación para reducir aquellos estilos de vida inadecuados en la población para prevenir el síndrome metabólico. La hipótesis a contrastar es que los estilos de vida como son el consumo de tabaco, alcohol, gaseosas, snacks, frutas, verduras y actividad física se relacionan con el síndrome metabólico en adultos y adultos mayores de la ciudad de Trujillo, 2019.

\section{MÉTODOS}

\section{Tipo y diseño de investigación}

De acuerdo al fin que se persigue, el presente estudio de investigación fue de tipo observacional, correlacional y transversal.

\section{Población y muestra}

La población estuvo constituida por los pobladores adultos de la ciudad de Trujillo. La muestra se determinó a través de la fórmula para población infinita considerándose una prevalencia de síndrome metabólico del $25,1 \%$, con un error correspondiente a $4,75 \%$, obteniéndose de esta manera 271 personas de 25 a 65 años ( 75 hombres y 196 mujeres), siendo 227 adultos ( 25 a 59 años) y 44 adultos mayores (60 a 65 años). La muestra estuvo conformada por 271 adultos, los cuales fueron divididos en dos grupos: 227 adultos jóvenes y 44 adultos mayores.

Fueron incluidos en este estudio adultos sanos, mientras que fueron excluidos aquellos adultos que presentaban enfermedades crónicas terminales (cáncer, cirrosis, insuficiencia renal crónica y enfermedad pulmonar obstructiva crónica), aquellos que presentaban enfermedades crónicas no transmisibles (diabetes, hipertensión y dislipidemias), deficiencia mental, y aquellos que no estuvieron en ayunas para los análisis bioquímicos. La selección de los participantes fue por conveniencia obtenida de los establecimientos de salud con mayor afluencia de usuarios: Los Jardines ${ }^{(87)}$, San Martin ${ }^{(86)}$, Hospital de Especialidades Básicas La Noria ${ }^{(51)}$ y Sagrado Corazón (47), todos del distrito de Trujillo. Posteriormente se les brindaron algunas recomendaciones específicas como la asistencia con ropa ligera para la evaluación antropométrica y estar en ayunas para la toma de muestra de sangre para evitar la alteración de los datos bioquímicos utilizados en el presente estudio.

\section{Instrumentos de recolección de datos}

Se aplicó un cuestionario de preguntas en la entrevista estructurada que permitió identificar los estilos de vida de 
cada participante. Este instrumento consistió en 7 ítems dirigidos al consumo o no de tabaco (cigarrillos), alcohol (cerveza pisco, ron), gaseosas, snacks (piqueos, chizitos y otros fritos), frutas (2 porciones al día), verduras (una porción al día) y si realiza actividad física un promedio de 30 minutos al día. Las bases teóricas para la elaboración de las preguntas del instrumento fueron las investigaciones realizadas por López et al. ${ }^{(12)}$ para el tabaco, Varela ${ }^{(13)}$ para la actividad física, Mirmiram et al. ${ }^{(14)}$ para las verduras, Mirmiram et al. ${ }^{(14)}$ y Sullivan et al. ${ }^{(15)}$ para las frutas, y Kant et al. ${ }^{(16)}$ para los snacks.

En el caso de los productos, cuando el participante respondió "nunca" o "muy esporádicamente" estas respuestas de intensidad baja se consideraron como "no consumidor", en tanto que si el participante respondió "frecuentemente" se consideró como "consumidor". Para el caso de la actividad física, al responder el participante "nunca" o "muy esporádicamente" se consideró que no realiza actividad física, en tanto al responder "frecuentemente", se consideró que si realiza después de 30 minutos al día. A continuación, se presenta el cuestionario de estilos de vida (ver Anexo).

Este instrumento consistió en 7 ítems dirigidos al consumo o no de tabaco (cigarrillos), alcohol (cerveza, pisco ron), gaseosas, snacks (piqueos, chizitos y otros fritos), frutas (2 porciones al día), verduras (una porción al día) y si realiza actividad física un promedio de 30 minutos al día. En el caso de los productos, cuando el participante respondió "nunca" o "muy esporádicamente", estas respuestas de intensidad baja se consideraron como "no consumo", en tanto que si el participante respondió "frecuentemente", se consideró como "si consume". Para el caso de la actividad física, al responder el participante "nunca" o "muy esporádicamente", se consideró que no realiza actividad física, en tanto al responder "frecuentemente", se consideró que sí lo realiza.

De esta manera, dicotimizaron las respuestas de la entrevista para facilitar la aplicación estadística chi cuadrado. El cuestionario fue validado por dos nutricionistas y un estadístico, aplicándose el test $V$ de Aiken ${ }^{(17)}$, siendo de valor 1. Luego de haber aplicado el cuestionario en un piloto de 20 personas, se determinó la confiabilidad con el test de Kuder Richardson, obteniéndose un coeficiente de 0,82 ; estableciéndose por consiguiente que el cuestionario es de buena apreciación. El cuestionario fue elaborado para ser respondido de manera rápida para cada uno de los estilos de vida, debido a que los participantes no podían permanecer mucho tiempo para poder cumplir con las actividades.

\section{Evaluación bioquímica y presión arterial}

Se realizaron pruebas bioquímicas y presión arterial con los participantes, con previa coordinación con los jefes de los establecimientos de salud. Los análisis se desarrollaron entre julio y agosto del 2019, en horario de 7 am a $10 \mathrm{am}$.

Se determinó la concentración de triglicéridos y colesterol HDL, con un monitor de colesterol "Mission 3 en 1" (Acon laboratories, San Diego, USA), para lo cual se desinfectó el dedo índice o medio con alcohol $96^{\circ}$, pinchándose con lanceta "Accu chek Safe T Pro Uno", se descartó la primera gota de sangre y se procedió a recolectar la muestra capilar en una micropipeta hasta alcanzar $30 \mu \mathrm{L}$, para luego verterla en la tira reactiva colocada previamente en el monitor de colesterol para su lectura. La muestra a utilizar para la medición de la glicemia se obtuvo por presión en el mismo dedo que se utilizó para la evaluación el perfil lipídico, colocándola en la tira reactiva previamente insertada en un glucómetro "Accu-Chek Performa Nano" (Roche Diabetes Care, Mannheim, Germany) y proceder con la lectura de la glicemia del paciente. Para la medición de la presión arterial, se empleó un tensiómetro digital "Ri Champion N" (Rudolf Riester, Jungingen, Alemania). Para ello, el participante se mantuvo en reposo aproximadamente 10 minutos, luego se solicitó que tome asiento, se le colocó el brazalete del tensiómetro, para realizar la medición.

\section{Evaluación antropométrica}

Para la evaluación antropométrica de la circunferencia de cintura se utilizó una cinta métrica metálica "Cescorf", se indicó a la persona ponerse de pie con los pies juntos, el torso desnudo y los brazos relajados a cada lado de su cuerpo, y se colocó la cinta métrica alrededor del cuerpo, desde el punto medio entre la última costilla y la cresta iliaca, para finalmente realizar la medición luego de una espiración normal. De acuerdo al consenso de la Asociación Latinoamericana de Diabetes (ALAD) ${ }^{(18)}$ : el perímetro normal para hombres es $<94 \mathrm{~cm}$ y para mujeres $<88 \mathrm{~cm}$.

\section{Diagnóstico de síndrome metabólico}

Para el diagnóstico de síndrome metabólico se necesitó considerar sólo 3 de los 5 criterios de riesgo propuestos por el ATP III armonizado y con los siguientes valores según sexo ${ }^{(18)}$ : Incremento del perímetro de cintura $\geq 94$ $\mathrm{cm}$ en hombres $\mathrm{y} \geq 88 \mathrm{~cm}$ en mujeres, triglicéridos $>150$ $\mathrm{mg} / \mathrm{dL}$ en ambos sexos, $\mathrm{HDL}<40 \mathrm{mg} / \mathrm{dL}$ en hombres o $<50 \mathrm{mg} / \mathrm{dL}$ en mujeres; en cuanto a la presión arterial elevada, tanto en hombres como mujeres: PAS $\geq 130$ $\mathrm{mmHg}$ o $P A D \geq 85 \mathrm{mmHg}$, y finalmente glicemia en ayunas $\geq 100 \mathrm{mg} / \mathrm{dL}$ en ambos sexos. 
Tabla 1. Estilos de vida en los pobladores adultos y adultos mayores de la ciudad de Trujillo, 2019

\begin{tabular}{|c|c|c|c|c|c|}
\hline \multirow{3}{*}{ Estilos de vida } & & \multicolumn{2}{|c|}{ Adultos } & \multicolumn{2}{|c|}{ Adultos mayores } \\
\hline & & \multicolumn{2}{|c|}{ (25-59 años) } & \multicolumn{2}{|c|}{ (60-65 años) } \\
\hline & & $\mathrm{fi}$ & $\%$ & $\mathrm{fi}$ & $\%$ \\
\hline \multirow{3}{*}{ Tabaco } & No consumidor & 208 & 91,6 & 41 & 93,2 \\
\hline & Consumidor & 19 & 8,4 & 3 & 6,8 \\
\hline & Total & 227 & 100,0 & 44 & 100,0 \\
\hline \multirow{3}{*}{ Alcohol } & No consumidor & 158 & 69,6 & 34 & 77,3 \\
\hline & Consumidor & 69 & 30,4 & 10 & 22,7 \\
\hline & Total & 227 & 100,0 & 44 & 100,0 \\
\hline \multirow{3}{*}{ Gaseosas } & No consumidor & 67 & 29,5 & 15 & 34,1 \\
\hline & Consumidor & 160 & 70,5 & 29 & 65,9 \\
\hline & Total & 227 & 100,0 & 44 & 100,0 \\
\hline \multirow{3}{*}{ Snacks } & No consumidor & 135 & 59,5 & 32 & 72,7 \\
\hline & Consumidor & 92 & 40,5 & 12 & 27,3 \\
\hline & Total & 227 & 100,0 & 44 & 100,0 \\
\hline \multirow{3}{*}{ Frutas } & No consumidor & 107 & 47,1 & 14 & 31,8 \\
\hline & Consumidor & 120 & 52,9 & 30 & 68,2 \\
\hline & Total & 227 & 100,0 & 44 & 100,0 \\
\hline \multirow{3}{*}{ Verduras } & No consumidor & 99 & 43,6 & 15 & 34,1 \\
\hline & Consumidor & 128 & 56,4 & 29 & 65,9 \\
\hline & Total & 227 & 100,0 & 44 & 100,0 \\
\hline \multirow{3}{*}{ Actividad física } & No realiza & 114 & 50,2 & 15 & 34,1 \\
\hline & SI realiza & 113 & 49,8 & 29 & 65,9 \\
\hline & Total & 227 & 100,0 & 44 & 100,0 \\
\hline
\end{tabular}

\section{Análisis de datos}

Para establecer la relación entre las variables se utilizó la prueba estadística del chi cuadrado, con un nivel de significancia de 0,05; en el programa estadístico Statistical Package for the Social Sciences (SPSS), versión 25.

\section{Aspectos éticos}

Se explicó a los participantes la importancia y objetivos del estudio, proporcionándoles un consentimiento informado escrito, en el que se indicó que los datos obtenidos se mantendrán en confidencialidad, con la potestad de hacer las consultas pertinentes, así como también de poder negarse a participar antes de firmar dicho documento sin exigencia alguna y siguiendo los principios establecidos en el Código de Ética de la Universidad César Vallejo, el cual se basa en los principios de la Declaración de Helsinki. La investigación fue aprobada por el Comité de Ética de la Universidad.

\section{RESULTADOS}

En la tabla 1 se observan los estilos de vida establecidos en los pobladores adultos y adultos mayores de la ciudad de Trujillo, donde $19(8,4 \%)$ y $3(6,8 \%)$ consumían tabaco; $69(30,4 \%)$ y 10 (22,7\%) consumían alcohol; 92 (40,5\%) y $12(27,3 \%)$ consumen snacks; $160(70,5 \%)$ y $29(65,9 \%)$ consumían gaseosas; $120(52,9 \%)$ y $30(68,2 \%)$ consumían frutas; $128(56,4 \%)$ y $29(65,9 \%)$ consumían verduras y por último $113(49,8 \%)$ y $29(65,9 \%)$ realizaban actividad física, respectivamente.

La tabla 2 reportó, por su parte la prevalencia del síndrome metabólico en adultos y adultos mayores siendo su frecuencia de $125(55,1 \%)$ y $32(72,7 \%)$, respectivamente, y de manera general, la prevalencia se estableció en $157(57,9 \%)$

Tabla 2. Prevalencia del síndrome metabólico en pobladores adultos y adultos mayores de la ciudad de Trujillo, 2019

\begin{tabular}{lccccc}
\hline \multirow{2}{*}{$\begin{array}{l}\text { Síndrome } \\
\text { metabólico }\end{array}$} & \multicolumn{2}{c}{ Adultos } & & \multicolumn{2}{c}{ Adultos mayores } \\
\cline { 2 - 3 } \cline { 5 - 6 } & $\mathbf{f i}$ & $\%$ & & $\mathbf{f i}$ & $\%$ \\
\hline No & 102 & 44,9 & & 12 & 27,3 \\
Sí & 125 & 55,1 & & 32 & 72,7 \\
Total & 227 & 100,0 & & 44 & 100,0 \\
\hline
\end{tabular}

${ }^{*}$ criterios del ATPIII armonizado ${ }^{(14)}$ 
En la tabla 3 se observa la relación entre los estilos de vida y el síndrome metabólico en adultos, donde el 43 $(18,9 \%)$ de los pobladores adultos que consumían snacks presentaron síndrome metabólico; en tanto que los que no consumían snacks y no presentaron síndrome metabólico representaron $53(23,3 \%),(p=0,037)$; mientras que 62 $(27,3 \%)$ pobladores adultos que no consumían verduras presentaron síndrome metabólico, en tanto que los que siempre consumían verduras y no presentaron síndrome metabólico representaron $65(28,6 \%),(p=0,044)$. Por lo tanto, existió relación significativa entre el consumo de snacks, verduras y el síndrome metabólico. No se observó relación entre consumo de tabaco, alcohol, gaseosas y actividad física con la presencia del síndrome metabólico con valores de $p>0,05$.

En la tabla 4 se halló la relación entre los estilos de vida y el síndrome metabólico en adultos mayores, donde se reportó que $24(54,5 \%)$ de los que no consumían snacks, presentaron síndrome metabólico; en tanto que 8 (18,2 \%) de los que consumían snacks y presentaron síndrome metabólico $(p=0,58)$, mientras que $10(22,7 \%)$ que no consumían verduras, presentaron síndrome metabólico; en tanto que $22(50 \%)$ que consumían verduras presentaron
Tabla 3. Relación entre los estilos de vida y el síndrome metabólico en pobladores adultos de la ciudad de Trujillo, 2019

\begin{tabular}{|c|c|c|c|c|c|}
\hline \multirow{3}{*}{$\begin{array}{l}\text { Síndrome } \\
\text { metabólico }\end{array}$} & \multicolumn{4}{|c|}{ Estilos de vida en adultos } & \multirow{3}{*}{$\begin{array}{c}\text { Significancia } \\
\text { (p) }\end{array}$} \\
\hline & \multicolumn{2}{|c|}{ No } & \multicolumn{2}{|c|}{ Sí } & \\
\hline & fi & $\%$ & fi & $\%$ & \\
\hline \multicolumn{6}{|c|}{ Tabaco } \\
\hline No & 95 & 41,9 & 7 & 3,1 & 0,459 \\
\hline Sí & 113 & 49,8 & 12 & 5,3 & \\
\hline Total & 208 & 91,7 & 19 & 8,4 & \\
\hline \multicolumn{6}{|c|}{ Alcohol } \\
\hline No & 75 & 33,0 & 27 & 11,9 & 0,245 \\
\hline Sí & 83 & 36,6 & 42 & 18,5 & \\
\hline Total & 158 & 69,6 & 69 & 30,4 & \\
\hline \multicolumn{6}{|c|}{ Gaseosas } \\
\hline No & 30 & 13,2 & 72 & 31,7 & 0,975 \\
\hline Sí & 37 & 16,3 & 88 & 38,8 & \\
\hline Total & 67 & 29,5 & 160 & 70,5 & \\
\hline \multicolumn{6}{|c|}{ Snacks } \\
\hline No & 53 & 23,3 & 49 & 21,6 & 0,037 \\
\hline Sí & 82 & 36,1 & 43 & 18,9 & \\
\hline Total & 135 & 59,4 & 92 & 40,5 & \\
\hline \multicolumn{6}{|c|}{ Frutas } \\
\hline No & 41 & 18,1 & 61 & 26,9 & 0,058 \\
\hline Sí & 66 & 29,1 & 59 & 26,0 & \\
\hline Total & 107 & 47,2 & 120 & 52,9 & \\
\hline \multicolumn{6}{|c|}{ Verduras } \\
\hline No & 37 & 16,3 & 65 & 28,6 & 0,044 \\
\hline Sí & 62 & 27,3 & 63 & 27,8 & \\
\hline Total & 99 & 43,6 & 128 & 56,4 & \\
\hline \multicolumn{6}{|c|}{ Actividad física } \\
\hline No & 51 & 22,5 & 51 & 22,5 & 0,952 \\
\hline Sí & 63 & 27,8 & 62 & 27,3 & \\
\hline Total & 114 & 50,2 & 113 & 49,8 & \\
\hline
\end{tabular}

Tabla 4. Relación entre los estilos de vida y el síndrome metabólico en pobladores adultos mayores de la ciudad de Trujillo, 2019

\begin{tabular}{|c|c|c|c|c|c|}
\hline \multirow{3}{*}{$\begin{array}{l}\text { Síndrome } \\
\text { metabólico }\end{array}$} & \multicolumn{4}{|c|}{ Estilos de vida en adultos mayores } & \multirow{3}{*}{$\begin{array}{l}\text { Significancia } \\
\text { (p) }\end{array}$} \\
\hline & \multicolumn{2}{|c|}{ No } & \multicolumn{2}{|c|}{ Sí } & \\
\hline & fi & $\%$ & $\mathrm{fi}$ & $\%$ & \\
\hline \multicolumn{6}{|c|}{ Tabaco } \\
\hline No & 10 & 22,7 & 2 & 4,5 & 0,112 \\
\hline Sí & 31 & 70,5 & 1 & 2,3 & \\
\hline Total & 41 & 93,2 & 3 & 6,8 & \\
\hline \multicolumn{6}{|c|}{ Alcohol } \\
\hline No & 8 & 18,2 & 4 & 9,1 & 0,334 \\
\hline Sí & 26 & 59,1 & 6 & 13,6 & \\
\hline Total & 34 & 77,3 & 10 & 22,7 & \\
\hline \multicolumn{6}{|c|}{ Gaseosas } \\
\hline No & 4 & 9,1 & 8 & 18,2 & 0,948 \\
\hline Sí & 11 & 25,0 & 21 & 47,7 & \\
\hline Total & 15 & 34,1 & 29 & 65,9 & \\
\hline \multicolumn{6}{|c|}{ Snacks } \\
\hline No & 8 & 18,2 & 4 & 9,1 & 0,580 \\
\hline Sí & 24 & 54,5 & 8 & 18,2 & \\
\hline Total & 32 & 72,7 & 12 & 27,3 & \\
\hline \multicolumn{6}{|c|}{ Frutas } \\
\hline No & 6 & 13,6 & 6 & 13,6 & 0,113 \\
\hline Sí & 8 & 18,2 & 24 & 54,5 & \\
\hline Total & 14 & 31,8 & 30 & 68,2 & \\
\hline \multicolumn{6}{|c|}{ Verduras } \\
\hline No & 5 & 11,4 & 7 & 15,9 & 0,516 \\
\hline Sí & 10 & 22,7 & 22 & 50,0 & \\
\hline Total & 15 & 34,1 & 29 & 65,9 & \\
\hline \multicolumn{6}{|c|}{ Actividad física } \\
\hline No & 3 & 6,8 & 9 & 20,5 & 0,436 \\
\hline Sí & 12 & 27,3 & 20 & 45,5 & \\
\hline Total & 15 & 34,1 & 29 & 65,9 & \\
\hline
\end{tabular}


síndrome metabólico $(p=0,516)$; por lo cual no existió relación entre el consumo snacks y el consumo de verduras con la presencia del síndrome metabólico. Así mismo, se observó que no existió relación entre consumo de tabaco, alcohol, gaseosas y actividad física con la presencia del síndrome metabólico con valores $p>0,05$.

\section{DISCUSIÓN}

Respecto al consumo de productos ultra procesados (gaseosas y snacks) consumidos por los participantes en el presente estudio (ver Tabla 1), estos afectarían al estado nutricional, dando como resultado una ganancia de peso, debido a que los patrones dietarios no saludables, de fácil acceso a alimentos de elevadas calorías, que se consumen fuera del horario de las comidas ${ }^{(19)}$, establecen el sobrepeso u obesidad que contribuye a que este grupo sea propenso a sufrir enfermedades crónicas no transmisibles. En un estudio similar desarrollado entre los años 2014 y 2016 en muestras poblacionales urbanas a nivel del mar y altura del Perú, en adultos de 18 a 65 años, se reportó que el $23 \%$ de la población adulta de Puno consumía snacks al igual que en el $28 \%$ en Cerro Pasco, $28 \%$ en Lima y $48 \%$ en lquitos, mientras que el consumo de gaseosas era del $53 \%$ en Puno, 48 \% en Cerro de Pasco, 56 \% en Lima y $82 \%$ en lquitos; resultados elevados como los encontrados en la ciudad de Trujillo, y que nos muestran la tendencia hacia una inadecuada alimentación en la población adulta peruana ${ }^{(20)}$.

En relación al síndrome metabólico observado en los pobladores de la ciudad de Trujillo, debe ser considerado con preocupación para la esperanza de vida media. En recientes décadas, el crecimiento económico causó grandes cambios en los patrones de estilos de vida en las sociedades modernas, lo cual se ha dirigido al desarrollo de los componentes clave del síndrome metabólico. Aunque no se muestran en los resultados, el síndrome metabólico identificado en los participantes se relaciona principalmente con la obesidad abdominal presente en el $81,92 \%$, y luego los otros factores como HDL bajo $33,95 \%$; hipertrigliceridemia 32,84\%; hiperglicemia $24,35 \%$ y, finalmente, la presión arterial alta o con tratamiento antihipertensivo que representa el 21,77\%.

El desarrollo de la obesidad está relacionado con el incremento de ácidos grasos libres, los cuales pueden bloquear la vía de señalización de la insulina ${ }^{(21)}$ y, a su vez, promover la producción del factor de necrosis tumoral (TNF a), interleuquina 6 (IL-6), entre otras citocinas, por parte de los macrófagos infiltrados en el tejido adiposo presente en el estado inflamatorio de la obesidad ${ }^{\text {(22-23). }}$
El factor de necrosis tumoral (TNF a) inhibe al sustrato del receptor de la insulina-1 (IRS-1), disminuyendo la amplificación intracelular de la señal del receptor insulínico ${ }^{(24-26)}$. Así mismo, los ácidos grasos libres pueden reducir la producción de óxido nítrico mediado por la insulina en las células endoteliales de los vasos sanguíneos que conduce a la hipertensión ${ }^{(27)}$.

Del mismo modo, la adiponectina se encuentra disminuida durante la obesidad, con lo cual se reducen sus propiedades de ayudar a determinadas células en su sensibilidad a la insulina y en el catabolismo de los ácidos grasos ${ }^{(28-29)}$; razones que conllevan diabetes y dislipidemia.

La relación significativa que existe entre el consumo de snacks (chizitos, fritos indicados en el estudio) y el síndrome metabólico, en el presente estudio resultó similar a lo reportado por otra investigación que concluyó que el consumo de snacks saludables contribuye a la obesidad abdominal e hipertensión en adultos con peso normal en el sudeste de Irán ${ }^{(30)}$.

Los snacks son productos que pueden alcanzar, en relación a su peso, entre 25 a $38 \%$ en grasa al final de su elaboración ${ }^{(31)}$, conteniendo ácidos grasos trans que están involucrados en el aumento de la concentración plasmática de lipoproteínas de baja densidad y reducen la concentración de las de alta densidad; además, también contribuyen con la obesidad e hipertensión (31,32), factores de riesgo del síndrome metabólico. Así mismo, el glutamato monosódico (GMS) que se usa como un potenciador del sabor en los snacks, es considerado un disruptor endocrino de la señal hipotalámica de la leptina ${ }^{(33)}$ que triplica la cantidad de insulina que el páncreas crea, causando problemas de diabetes mellitus tipo Il y obesidad ${ }^{(34)}$.

Sin embargo, ya sea el consumo de snacks u otro producto hipercalórico, como los elaborados a base de harinas, potencian el problema del bajo consumo de verduras observado en el presente estudio, ya que la fibra es esencial en el incremento de receptores para LDL hepáticos, lo que permite reducir los niveles plasmáticos de LDL y a su vez, se encuentra asociado con el incremento de la concentración de la lipoproteína $\mathrm{HDL}^{(35,36)}$, esencial en la prevención de los procesos aterogénicos y que, en el caso del síndrome metabólico, su concentración se encuentra disminuida.

En esta investigación no se encontró relación entre el tabaquismo, la actividad física, consumo de alcohol y de bebidas azucaradas con el síndrome metabólico en 
adultos y adultos mayores, difiriendo de otros estudios que encontraron al tabaquismo como principal factor de riesgo del síndrome metabólico ${ }^{(37)}$ y también porque que considera tanto al consumo de alcohol como de tabaco y al ejercicio físico ${ }^{(10)}$. Lo encontrado se debe posiblemente a otros factores relacionados con la edad, las modificaciones hormonales, tanto en mujeres como en hombres, o el estrés oxidativo ${ }^{(38)}$; aspectos no evaluados en el presente estudio.

El síndrome metabólico es un conjunto de alteraciones presentes en diferentes sistemas orgánicos, con amplias variaciones fenotípicas en personas con una predisposición endógena, determinada genéticamente y condicionada por factores ambientales ${ }^{(39)}$. Se han determinado factores genéticos específicos de riesgo que se asocian con la predisposición del síndrome metabólico, como es el caso del gen asociado a la obesidad de la masa grasa, conocido como gen FTO (del inglés: fat mass and obesity-associated protein); aspectos a considerar de manera conjunta con los estilos de vida en futuros estudios multivariados ${ }^{(40)}$.

En el caso de este estudio de corte transversal, no se garantiza la causalidad del consumo de snacks y el bajo consumo de verduras en el síndrome metabólico; incluso variando la modalidad del cuestionario a aplicar, ya sea través de un recordatorio de hábitos de consumo por cantidades (por día o semana) o por las actividades en un momento dado, ya que es posible una persona no recuerde, más aun si los participantes asistieron a la consulta externa en cada establecimiento de salud y teniendo en cuenta el tiempo de sus actividades inherentes podría tener influencia en sus respuestas. Es más probable que un registro de dichos estilos de vida en una línea de tiempo ayudaría a corroborar lo encontrado a través de un estudio de cohortes.

Finalmente se concluye indicando que existe relación significativa entre el consumo de snacks y verduras con el síndrome metabólico en los adultos, mientras que no se encontró relación entre los estilos de vida con el síndrome metabólico en adultos mayores de la ciudad de Trujillo.

\section{REFERENCIAS}

1. Mozzafari H, Namazi N, Larijani B, Bellissimo N, Azadbakht $\mathrm{L}$. The association of dietary acid load with cardiovascular risk factors and the prevalence of metabolic syndrome in Iranian women: A cross-sectional study. Nutrition [Internet]. 2019 [Consultado 2019 Nov 16]; 67(1): 1-7. Disponible en: https://pubmed.ncbi.nlm.nih. gov/31568970/

2. De Caluwé L, Van Buitenen N, Gelan P, Crunelle C, Thomas R, Casseres $\mathrm{S}$, et al. Prevalence of metabolic syndro- me and its associated risk factors in an African-Caribbean population with severe mental illness. Psychiatry Research. [Internet]. 2019 [Consultado 2019 Nov 16]; 281(1): 1-10. Disponible en: https://europepmc.org/article/med/31546147

3. Dussaillant C, Echeverría G, Villarroel L, Marin P, Rigotti A. Una alimentación poco saludable se asocia a mayor prevalencia de síndrome metabólico en la población adulta chilena: estudio de corte transversal en la Encuesta Nacional de Salud 2009-2010. Nutr Hosp [Internet]. 2015 [Consultado 2019 Nov 16]; 32(5): 2098-2104. Disponible en: http://scielo.isciii.es/pdf/nh/v32n5/27originalsindromemetabolico01.pdf

4. Fortino M, Zurbriggen A, Botto C, Giangrossi G. Síndrome metabólico: prevalencia de factores asociados a la dieta y al estilo de vida en una población de riesgo. Revista bioquímica y patología clínica. [Internet]. 2007 [Consultado 2019 Nov 16]; 71(3): 24-28. Disponible en: http://www.redalyc.org/pdf/651/65112133004.pdf

5. Giuseppe S, Dianin M, Bertocco A, Zanforlini B, Curreri C, Mazzochin M. Gender differences in the impact of metabolic syndrome components on mortality in older people: A systematic review and meta-analysis. Nutrition, Metabolism \& Cardiovascular Diseases [Internet]. 2020 [Consultado 2020 Nov 16]; 30(9): 1452-1464. Disponible en: https://www.sciencedirect.com/science/article/abs/ pii/S0939475320301575

6. Saklayen M. The Global Epidemic of the Metabolic Syndrome. Current Hypertension Reports [Internet]. 2018 [Consultado 2020 Nov 16]; 20(2): 12. Disponible en: https://www.ncbi.nlm.nih.gov/pmc/articles/ PMC5866840/

7. Arsentales $\mathrm{V}$, Tenorio $\mathrm{M}$, Bernabé $\mathrm{A}$. Asociación entre actividad física ocupacional y síndrome metabólico: Un estudio poblacional en Perú. Rev Chil Nutr. [Internet]. 2019 [Consultado 2019 Nov 16]; 46(4): 392-399. Disponible en: https://scielo.conicyt.cl/scielo.php?script=sci_abstract\&pid=S0717-75182019000400392\&lng=pt\&nrm=iso

8. Linares-Reyes E, Castillo-Minaya K, Ríos-Mino M, Huamán-Saavedra J. Estudio de correlación entre los diagnósticos de cintura hipertrigliceridémica y síndrome metabólico en adultos de Trujillo, Perú. Rev Peru Med Exp Salud Pública [Internet]. 2014 [Consultado 2019 Nov 16]; 31(2): 254-60. Disponible en: http:// www.scielo.org.pe/scielo.php?script=sci_arttext\&pi$d=S 1726-46342014000200009$

9. Vicente M, López A, Ramírez M, Capdevila L, Terradillos M, Aguilar E. Parámetros de riesgo cardiovascular, síndrome metabólico y consumo de alcohol en población laboral. Endocrinol Nutr. [Internet]. 2015 [Consultado 2019 Nov 16]; 62(4): 161-7. Disponible en: https://www. elsevier.es/es-revista-endocrinologia-nutricion-12-articulo-parametros-riesgo-cardiovascular-sindrome-metabolico S1575092215000273

10. Vallejos R. Acerca del artículo: Factores de riesgo asociados al síndrome metabólico en conductores del transporte público en Cochabamba, Bolivia. Gac Med Bol. [Internet]. 2019 [Consultado 2019 Nov 16]; 42(1): 84-85. Disponible en: http://gacetamedicaboliviana.com/index.php/gmb/article/view/69

11. Bassi N, Karagodin I, Wang S, Vassallo P, Priyanath A, Massaro E, et al. Lifestyle Modification for Metabolic Syndrome: A Systematic Review. The American Journal 
of Medicine [Internet]. 2016 [Consultado 2019 Nov 16]; 127(12): 1242e1-1242e10. Disponible en: https://pubmed.ncbi.nlm.nih.gov/25004456/

12. López AA, Rivero Yl, Vicente MT, Gil M, Tomás M, y Riutord Índices aterogénicos en trabajadores de diferentes sectores laborales del área mediterránea española. Clínica e Investigación en Arteriosclerosis [Internet]. 2014 [Consultado 2021 May 19]; 27(3): 118-128. http://dx.doi. org/10.1016/j.arteri.2014.10.004

13. Varela M, Duarte C, Salazar I, Lema L, Tamayo J. Actividad física y sedentarismo en jóvenes universitarios de Colombia: Prácticas, motivos y recursos para realizarlas. Revista Colombia Médica [Internet]. 2011 [Consultado 2021 May 19]; 42(3): 269-277. Disponible en: https:// www.redalyc.org/pdf/283/28322503002.pdf

14. Mirimiran P, Bakhshi B, Hosseinpour-Niazi S, Sarbazi N, Hejazi J, Azizi F. Does the association between patterns of fruit and vegetables and metabolic syndrome incidence vary according to lifestyle factors and socioeconomic status? Nutrition, Metabolism \& Cardiovascular Diseases [Internet]. 2020 [Consultado 2021 May 19]; 30(8): 1322-1336. Disponible en: https://www.sciencedirect.com/science/article/abs/pii/S0939475320301290

15. Sullivan V, Na M, Proctor D, Penny M, Ehterton K, Petersen K. Consumption of Dried Fruits Is Associated with Greater Intakes of Underconsumed Nutrients, Higher Total Energy Intakes, and Better Diet Quality in US Adults: A Cross-Sectional Analysis of the National Health and Nutrition Examination Survey, 2007-2016. Journal Academic Nutrition Dietetic [Internet]. 2020 [Consultado 2021 May 19]; 121(7): 1258-1272. https://doi.org/10.1016/j. jand.2020.08.085

16. Kant A. Eating patterns of US adults: Meals, snacks, and time of eating. Physiology \& Behavior [Internet]. 2018 [Consultado 2021 May 22]; 193 (Pt B): 270-278. doi:10.1016/j.physbeh.2018.03.022

17. Robles B. Índice de validez de contenido: Coeficiente $V$ de Aiken. Pueblo Cont. [Internet]. 2018 [Consultado 2018 Oct16]; 29(1): 193-7. Disponible en: http://journal. upao.edu.pe/PuebloContinente/article/view/991

18. Lizarzaburu J. Síndrome Metabólico: Concepto y aplicación práctica. An Fac med [Internet]. 2013 [Consultado 2018 Oct 16]; 74(4): 315-20. Disponible en: http:// www.scielo.org.pe/scielo.php?script=sci_arttext\&pi$\mathrm{d}=\mathrm{S} 1025-55832013000400009$

19. Mirzababaei A, Sajjadi S, Ghodoosi N, Pooyan S, Arghavani $H$, Yekaninejad $M$, et al. Relations of major dietary patterns and metabolically unhealthy overweight/ obesity phenotypes among Iranian women. Diabetes \& Metabolic Syndrome: Clinical Research \& Reviews [Internet]. 2019 [Consultado 2020 Oct16]; 13(1): 322-331. doi: 10.1016/j.dsx.2018.09.012

20. Caballero L. Patrones de consumo alimentario, estado nutricional y características metabolómicas en muestras poblacionales urbanas del nivel del mar y altura del Perú [Internet] Lima: Universidad Peruana Cayetano Heredia; 2017 [Consultado 202105 20]. Disponible en: http://repositorio.upch.edu.pe/handle/upch/1012

21. Roura-Guiberna A, Jiménez-Mena R y Olivares-Reyes J. El papel de los ácidos grasos libres en la resistencia a la insulina. Gac Med Mex. [[Internet]. 2017 [Consultado 2020 Oct16]; 153(7): 852-863. Disponible en: https://gacetamedicademexico.com/frame_esp.php?id=86
22. Sierra J, Gómez L. Asociación de los niveles de adiponectina y del factor de necrosis tumoral-alfa (TNF-a) con la albuminuria en pacientes con diabetes mellitus tipo 2. Medicina y Laboratorio [Internet]. 2017 Consultado20200ct16]; 23(5): 257-270. Disponible en: https://www.medigraphic.com/pdfs/medlab/myl-2017/ myl175-6e.pdf

23. Figueiredo PS, Inada AC, Marcelino G, Cardozo CML, Freitas $\mathrm{K}$ de $\mathrm{C}$, Guimarães $\mathrm{R}$ de $\mathrm{CA}$, et al. Fatty acids consumption: The role metabolic aspects involved in obesity and its associated disorders. Nutrients [Internet]. 2017 [Consultado 2020 Oct 16]; 9(10):1-32. Disponible en: https://www.mdpi.com/2072-6643/9/10/1158/htm

24. Ramírez M, Sánchez C. El factor de necrosis tumoral-a, la resistencia a la insulina, el metabolismo de lipoproteínas y la obesidad en humanos. Nutr Hosp. [Internet]. 2012 [Consultado 2019 Oct 16]; 27(6): 1751-1757. Disponible en: http://scielo.isciii.es/pdf/nh/v27n6/01articuloespecial01.pdf

25. Ros M, Medina G. Obesidad, adipogénesis y resistencia a la insulina. Endocrinología y Nutrición [Internet]. 2011 [Consultado20190ct16]; 58(7): 360-369. Disponible en: https://www.elsevier.es/es-revista-endocrinologia-nutricion-12-articulo-obesidad-adipogenesis-resistencia-insulina-S157509221100218X

Cooke AA, Connaughton RM, Lyons CL, McMorrow AM, Roche HM. Fatty acids and chronic low grade inflammation associated with obesity and the metabolic syndrome. Eur J Pharmacol [Internet]. 2016 [Consultado 2019 Oct 16]; 785: 207-14. Disponible en: http://dx.doi. org/10.1016/j.ejphar.2016.04.021

26. Ghosh A, Gao L, Thakur A, Siu PM, Lai CWK. Role of free fatty acids in endothelial dysfunction. J Biomed Sci. [Internet]. 2017 [Consultado20190ct16]; 24(1): 1-15. Disponible en: https://pubmed.ncbi.nlm.nih.gov/28750629/

27. Gómez P, Alarcón A, Rodríguez F, Díaz E. La adiponectina como blanco terapéutico. Med Int Méx. [Internet]. 2017 [Consultado20190ct16]; 33(6): 770-777. Disponible en: https://www.medigraphic.com/pdfs/medintmex/mim2017/mim176i.pdf

28. Medina-Urrutia A, Posadas-Romero C, Posadas-Sánchez $\mathrm{R}$, Jorge-Galarza E, Villarreal-Molina T, González-Salazar $M$ del $C$, et al. Role of adiponectin and free fatty acids on the association between abdominal visceral fat and insulin resistance. Cardiovasc Diabetol. [Internet]. 2015 [Consultado 2019 Oct 16]; 14(1): 1-8. Disponible en: https://cardiab.biomedcentral.com/articles/10.1186/ s12933-015-0184-5

29. Zare J, Niknejad N, Shahbazian H, Latifi SM, Niknejad B, Hormoznejad R. The Relationship between Snacking and Risk of Individual Components of Metabolic Syndrome in Normal-weight Adults: A Cross-sectional Study. JNFS [Internet]. 2017 [Consultado 2020 Nov 20]; 2(4): 265-278. Disponible en: http://jnfs.ssu.ac.ir/article1-110-en.pdf

30. Gonzales JDM. Determinación del contenido de ácidos grasos cis, trans, saturados, monoinsaturados y poliinsaturados en snacks mediante cromatografía de gases [Internet] Chiclayo: Universidad Nacional Pedro Ruiz Gallo; 2020 [Consultado 2021 May 08] Disponible en: http:// repositorio.unprg.edu.pe/handle/UNPRG/8410

31. Bouchon P. Understanding Oil Absorption During DeepFat Frying. En: Taylor S. Advances in Food and Nutrition 
Research [Internet] Lincoln: Academic Press; 2009: 209233 [Consultado 2019 Nov 20] Disponible en: https:// pubmed.ncbi.nlm.nih.gov/19595388/

32. Carbonero M. Glutamato monosódico “La trampa de los alimentos sabrosos". Trastornos de la Conducta Alimentaria [Internet]. 2013 [Consultado 2019 Nov 20]; 17(1): 1863-1876. Disponible en: https://www.tcasevilla.com/ archivos/glutamato_monosodico_y_obesidad.pdf

33. Ardura P. Relación entre obesidad y disruptores endocrinos. Revista para profesionales de la salud [Internet]. 2019 [Consultado 2019 Nov 20]; 2(18):44-71. Disponible en: https://www.npunto.es/revista/18/relacion-entre-obesidad-y-disruptores-endocrinos

34. Babio N, Balanza R, Basulto J, Bullo M, Salas J. Dietary fibre: influence on body weight, glycemic control and plasma cholesterol profile. Nutr Hosp. [Internet]. 2010 [Consultado 2018 Nov 20]; 25(3): 327-340. Disponible en: https://pubmed.ncbi.nlm.nih.gov/20593113/

35. Mataix J. Nutrición y Alimentación Humana. Nutrientes y Alimentos [Internet]. Barcelona: Oceáno/Ergon; 2005. [Consultado 2018 Nov 20]. Disponible en: https://ergon.es/ producto/nutricion-y-alimentacion-humana-2a-edicion/

36. Trujillo B, Trujillo E, Trujillo $M$, Brizuela C, Garcia $M$, Gonzalez $\mathrm{M}$, et al. Frecuencia del síndrome metabóli- co y factores de riesgo en adultos con y sin diabetes mellitus e hipertensión arterial. Rev. Salud Pública [Internet]. 2017 [Consultado 2018 Nov 20]; 19(5): 609-616. Disponible en: https://www.redalyc.org/ pdf/422/42255435004.pdf

37. Zafon C. Envejecimiento más allá del síndrome metabólico. Rev Esp Geriatr Gerontol. [Internet]. 2007 [Consultado 2018 Nov 20]; 42(5): 302-311. Disponible en: https://www.elsevier.es/es-revista-revista-espanola-geriatria-gerontologia-124-articulo-envejecimiento-resistencia-insulina-mas-alla-S0211139X07735667

38. Navia M, Yaksic N, Aguilar X, Farah J, Chambi E, Mollinedo E. Factores de riesgo asociados a síndrome metabólico en población habitante de 3600 y 4100 m.s.n.m. Rev Med La Paz [Internet]. 2015 [Consultado 2018 Nov 20]; 21(2): 6-17. Disponible en: http://www.scielo.org.bo/ $\mathrm{pdf} / \mathrm{rmcmlp} / \mathrm{v} 21 \mathrm{n} 2 / \mathrm{v} 21 \mathrm{n} 2 \_a 02 . p d f$

39. Osman W, Khan S, Jelinek H, Almahmeed W, Tay G, Alsafar $\mathrm{H}$. Clinical correlations and genetic associations of metabolic syndrome in the United Arab Emirates. Gene. [Internet]. 2020 [Consultado 2020 Nov 04]; 738(1): 1-8. Disponible en: https://researchoutput.csu.edu.au/en/ publications/clinical-correlations-and-genetic-associations-of-metabolic-syndr 